\title{
VR-Enabled Chatbot System Supporting Transformer Mass-Customization Services
}

\author{
Finch C.-T. WUª, Oscar N.-J. HONG ${ }^{\mathrm{a}}$, Amy J.C. TRAPPEY ${ }^{\mathrm{a}}$, Charles V. TRAPPEY ${ }^{\mathrm{b}}$ \\ ${ }^{a}$ Department of Industrial Engineering and Engineering Management, National Tsing \\ Hua University, Hsinchu, Taiwan \\ ${ }^{b}$ Department of Management Science, National Chiao Tung University, Hsinchu, Taiwan
}

\begin{abstract}
Chatbot is a conversational question answering (Q\&A) system capable of natural language communication between a computer system and a person. The use of chatbots for 24-hour customer service provides quick responses that solve problems online. This approach is quickly becoming a convenient way for companies to enhance their customer services without location or knowledgeable staff limitations. This research proposes a system framework and develops a prototype virtual reality (VR) enabled transformer mass-customization consultation chatbot. The chatbot technique is a retrieval-based intelligent system. First, thousands of transformer specific frequently asked questions (FAQs) are collected as a Q\&A dataset for technical supports retrieval. More than 1.2 million engineering Wikipedia pages and engineering technical papers are used to train a word embedding model used for natural language processing and question-answer retrieval. The chatbot is integrated into a virtual reality (VR) immersive user interface (UI) environment enabling users to make transformer design changes while querying the system about specifications and standards while interacting with 3D models from the company's knowledge base archive. The system provides two unique UIs for personal computer (PC) and a helmet-based immersive interface. The system supports real-time consultation of mass-customized transformers and their bills of materials (BOM) for design review, analysis and cost estimation.
\end{abstract}

Keywords. Intelligent chatbot, virtual reality, smart manufacturing, electric transformers

\section{Introduction}

Intelligent chatbots have been developed using artificial intelligence and natural language processing and thousands of chatbots are actively used globally [1]. Virtual reality is essential for engineering support. Using VR applications, a chatbot can be integrated into an immersive environment to enhance interactions between chatbot knowledge systems and users. At present, chatbots are used for online services in ecommerce and service industries. For engineering or manufacturing firms, chatbot development faces challenges due to the technology-specific knowledge required to build sophisticated engineering linguistic consultation. For example, power transformers are engineering assets that are critical machineries used in electric power distribution. Because of the high customization required in power transformer design and manufacturing, a smart consultation agent (or chatbot) that can communicate and answer technical questions effectively must understand the company's knowledge base as well as the customers' requirements. People often spend a lot of time searching for answers 
to engineering-related issues because of the lack of specific domain knowledge or the mismanagement of creating knowledge-based systems to store the intangible assets of the firm. There is a need for an interface where the manufacturers can interact with their customers, answer technical FAQs in real time, and re-use elements of design and engineering that are proprietary to the firm. Therefore, this research aims to integrate the consultation chatbot with a VR environment for transformer mass-customization Q\&As which automatically and accurately provide knowledgeable technical support to customers at a delivery rate that surpasses existing methods.

\section{Literature review}

In this section, the literature of related methodologies in VR and chatbot technologies are reviewed. Chatbot, also known as talkbot or service agent, is a software that creates user friendly conversations with users via text or voice. Chatbots can deliver real-time service to customers and intelligently simulate the communication between the system and user [2]. Based on different design techniques, chatbots are classified rule-based chatbots and artificial Intelligence (AI)-based chatbots. AI-based chatbots are classified as retrieval-based chatbots and generative-based chatbots [3]. Rule-based chatbots are constructed using pre-set scripted dialogs. The chatbot will provide answers according to the logic of the known domain. The techniques used in ruled-based chatbot are less intelligent and lack interaction. The benefit is that the construction cost is low and acceptable results can be realized if the script is written sufficiently and creatively [1]. Retrieval-based methods use natural language processing techniques to efficiently select proper responses from existing conversational data and generates responses using natural language generation models trained by conversation data [4].

As an intangible asset, patent documents are widely used in business. Through patent analysis, there are many methods to structure and understand the specific knowledge domain using algorithms. Hong, et al. [5] present an advanced approach to generate a patent map which is called the Technology Function Product Matrix (TFPM). The research uses the generated matrix to present the analysis of dialog system technology in chatbots and provides the hotspot distributions of key technologies, functional improvements, and novel applications. Table 1 shows five patent developments related to chatbot dialog systems. Chatbot related patents tend to focus on intent detection and domain identification. Intent detection is a technology which uses text mining algorithms and detection rules to detect user intention. The results show embedded device and natural language understanding are the current technology developments. NL understanding is a method where systems assume what a speaker actually means. Further product application applies dialogue function analysis to an embedded system. From the discussion of identified technology trends, the chatbot related technologies are shown in Table 1. 
Table 1. Top 5 patent hotspots of dialog system in chatbots.

\begin{tabular}{ll}
\hline Technology sub-system/ function or product & Patent count \\
\hline Intent detection (T22)/ Embedded device (P3) & 141 \\
\hline Domain identification (T21)/ Embedded device (P3) & 122 \\
\hline Intent detection (T22)/ NL understanding (F1) & 114 \\
\hline Content planning (T41)/ Embedded device (P3) & 113 \\
\hline Domain identification (T21)/ NL understanding (F1) & 109
\end{tabular}

Virtual reality (VR) technology enables users to have the feeling of being present in the environment and creates a virtual interaction with the human interacting with the system [5]. For manufacturing industries, virtual reality has been applied for many years and was first reported to as a simulation tool in the 1960s [6]. The advances of virtual reality (VR) or augmented reality (AR) technologies have provided different engineering applications, such as manufacturing planning, process simulation, product design and testing, modelling, and employee training [7]. For example, Gonzalez-Badillo et al. [8] present a virtual reality haptic enabled platform. The platform provides an environment to design, plan, and evaluate virtual manufacturing assemblies of components while allowing engineers to manipulate and interact with virtual components in real-time. Peng et al. [9] present a VR system for interactive modular fixture configuration design and apply a multi dimension modular fixture assembly model to enhance visualization and management. The proposed 3D manipulation approach improves intuitive interaction and more accurately positions components in the virtual environment which is then transferred to actual manufacturing.

\section{Methodology}

In this section, the method and framework of three sub-systems are introduced: chatbot (question answering system), cost estimation system, and the virtual reality environment. In the first sub-system, the FAQ data collection, data preprocessing process, and information retrieval for the question answering system are proposed. The second part explains the methods of building a knowledge base to store key information of power transformers and cost estimations based on previous Bills of Material (BOM). For the section describing the virtual reality engine, modeling software building $3 \mathrm{D}$ models, the development and execution environment in Unity, scene design, objects control, and applications on different platforms are described. The framework of the proposed consultation system is shown in Figure 1, and details of the system are discussed in the following sections. 


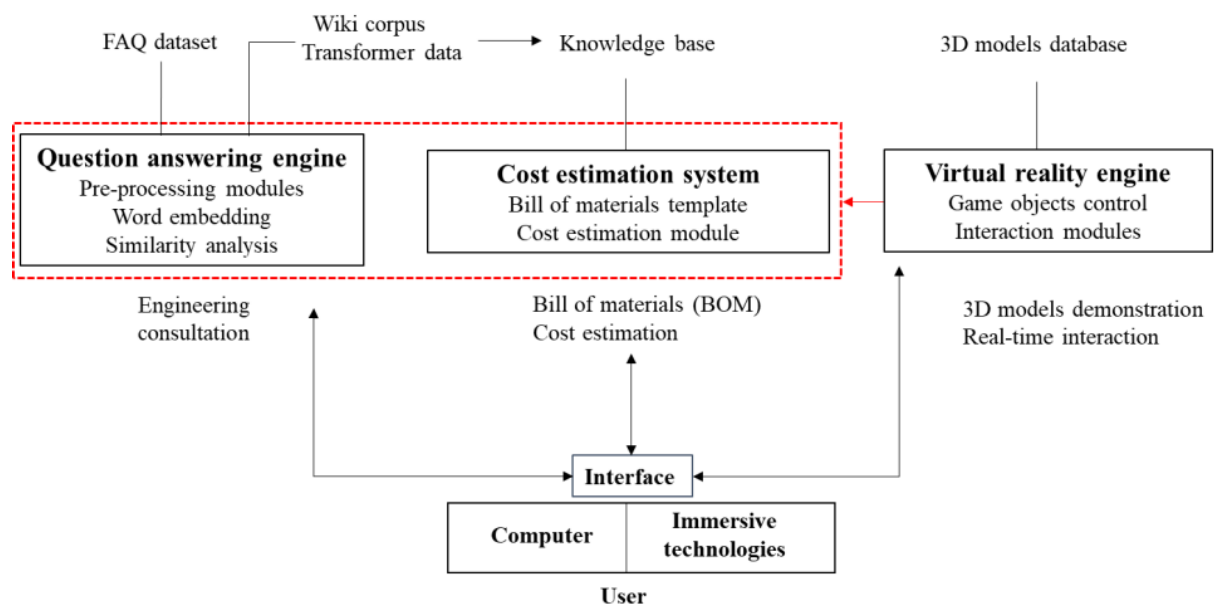

Figure 1. Framework of VR enabled chatbot for a power transformer manufacturer.

\subsection{Chatbot (Q\&A system)}

The computerized question and answering system provide users with a way to communicate in natural language receive answers instead of web pages. The design technique of the chatbot in this research is to use a retrieval-based similarity calculation in response to a selection from the FAQ (Frequently Asked Questions) conversation data set.

The retrieval-based question answering system allows users to ask questions in natural language and retrieve answers from a large number of heterogeneous materials accurately and quickly. The chatbot eliminates the process of reorganizing the answers and improves the efficiency of the systems accuracy of answers. The FAQ collection benefits the similarity between the user question and the questions in the data set with returning the best result to the user. The process of selecting an answer in the question answering system in this research can be divided into the following steps and the flowchart of the system is shown in Figure 2.

1. Pre-process questions to remove non-informative words and save computing time.

2. Derive word vectors and calculate the similarity between user's question and each question in FAQ dataset. The answer corresponding to the question with the highest similarity is returned to user.

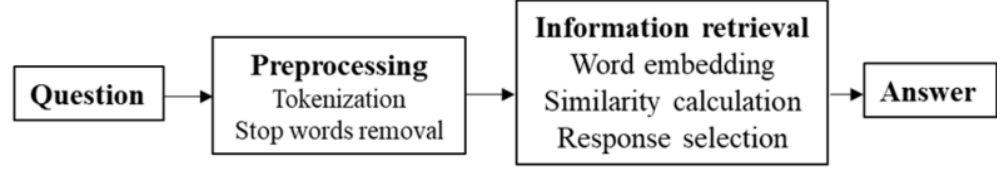

Figure 2. Flowchart of question answering.

The tool used to train a word embedding model is called Word2Vec [10]. Word2vec is a prediction-based model that predicts adjacent words. The word vector is part of the model parameters, and the best word vector model is learned during model training. After deriving the vectors of every word in a question sentence, the similarity calculation between the user's question and questions in dataset is conducted. The cosine angle is used to calculate the similarity between the vectors, which is called cosine similarity. 
The value of each cosine similarity is between 0 and 1 . The larger the value, the higher the similarity of the two words. Cosine similarity works in vector spaces of any dimension, and is most commonly used in high-dimensional positive spaces, i.e. information retrieval. The formula of cosine similarity is:

$$
\text { Similarity }=\cos \theta=\frac{\sum_{\mathrm{i}=1}^{\mathrm{n}} \mathrm{Ai} * \mathrm{Bi}}{\sqrt{\sum_{\mathrm{i}=1}^{\mathrm{n}} \mathrm{Ai}^{2}} \sqrt{\sum_{\mathrm{i}=1}^{\mathrm{n}} \mathrm{Bi}^{2}}}
$$

$A i$ and $B i$ are components of vector $A$ and vector $B$ respectively.

\subsection{Cost estimation system}

Power transformers are the primary equipment of power plants and substations and are highly customized to satisfy customer needs. It is necessary that manufacturers and customers have honest communications when negotiating prices. By introducing the cost estimation sub-system, customers have a preliminary understanding of the price of the product. A Request for Quotation (RFQ) in the power transformer industry is a document used to request for bids from qualified manufactures of transformers. The RFQ document specifies the requirements of the transformer to be purchased. The requirements could be details such as how it will be constructed and designed, desired components or the standards to be followed. The first task to construct a cost estimation system is to build a knowledge base (KB). The knowledge base contains the estimated cost of all possible key materials and components of a transformer. These costs are compared to the identified key components with the KB, the BOM tables, and then the estimated costs are tabulated.

The Bill of Materials (BOM) generation enables the customer to acquire a tentative cost of the required transformer product when receiving a summary of RFQ document. The summary report contains specifications for the requested transformer such as capacity, voltage, and phase. These quotes are critical factors that determine product cost that enable manufacturers and customers flexibility in bargaining. Figure 3 shows the process of BOM generation. The information in RFQ summary will be compared with the knowledge base $(\mathrm{KB})$ to identify the key components and their required raw materials. All the extracted components will be decomposed to a list of required raw materials. Based on the material information, the estimated tentative cost of a transformer will be calculated. Once all the components in RFQ summary file have been compared, the list of components, materials, and total estimated cost will be used to generate a BOM and send back to the user in a virtual environment.

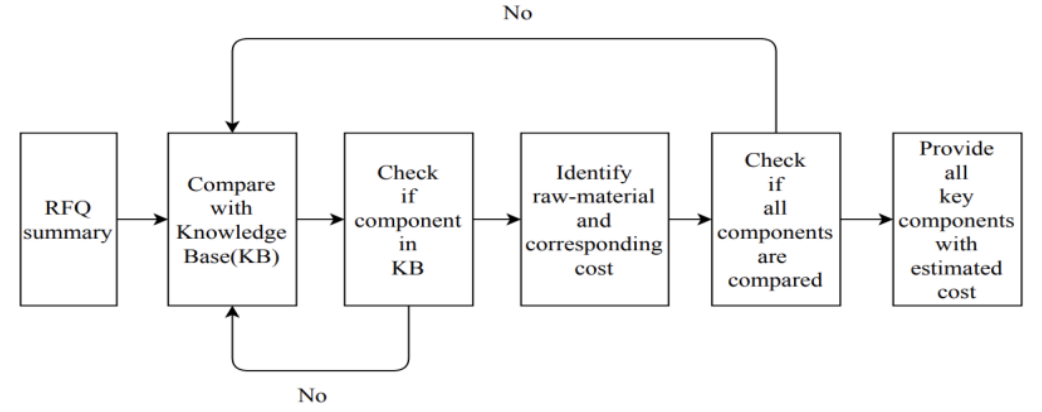

Figure 3. Flowchart of BOM generation. 


\subsection{Virtual reality environment}

Using the virtual reality (VR) environment, the consultation system provides a threedimensional (3D) transformer model to customer and also enables users to interact with the model. The system can operate on two different platforms. User can simply see the model and interact with the 3D objects using the mouse and keyboard of a computer. The second platform enables immersive technologies where the user can wear an HTC VIVE helmet and controller. Users can experience an immersive environment to see the model and have a clearer and more intuitive understanding of the product. The following sections introduce the modeling software used in this research and the development of Unity 3D game engine applications.

In this research, SolidWorks, the first Windows-based 3D CAD modeling software, is used in engineering design and provides a powerful platform for the design of components, especially the assembly of large components which are hard to visualize. SolidWorks simplifies assembly design and provides the same visualization as realworld, design-and-obtain, and allows users view, rotate, and even enter the interior of the equipment. Unity 3D is used to construct the user interaction environment.

Unity is a cross-platform game engine which enables users to create games, simulations and interactive experiences in both $2 \mathrm{D}$ and $3 \mathrm{D}$, and the engine offers a primary scripting API in C\#, for both the Unity editor in the form of plugins, and games themselves, as well as drag and drop functionality. The development environment of Unity can be divided into several parts: scene design interface, scene object structure, object structure, object list, object attribute, execution interface. When designing the user interface of the Unity program in this research, it is divided into three parts: the chatbot (dialogue window), model interaction area and the functions list. Chatbot (Dialogue window): Users can interact with the chatbot system within a dialogue window to conduct an engineering consultation in this virtual environment via a keyboard or microphone. Model interaction area: In this area, users can interact with 3D models in real time, for example, users can click on the key components (i.e. conservator, bushing, cooling system) to view relevant specification information and use the specified buttons or commands to disassemble the model to seeing the internal core structure. Functions list: The functions list enables users customize the components or specifications of models. Figure 4 summarizes the structure of functions in the immersive environment. The introductions of the interface and achieved functions are described in the Section 3.

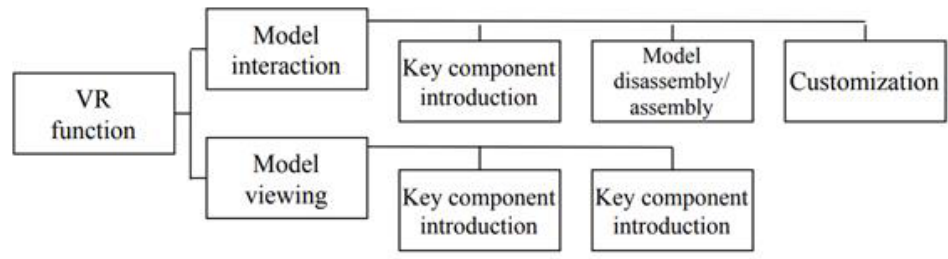

Figure 4. Structure of VR functions.

\subsection{Systems integration}

The previous sections discuss the methods and processes for constructing the three subsystems. This section discusses how to integrate question answering systems (chatbot) and cost estimation systems into a virtual reality environment. The first subsystem includes a preprocessing module, a word embedding model, and a similarity calculation 
module. The research uses Python to implement tasks of natural language processing. Python provides a rich Natural Language Toolkit library to help with pre-questioning, word vector model training. We utilize the trained model and load the model with the package in .NET Framework so that the retrieval-based system can be accessed in the virtual reality development software Unity. Since the chatbot is integrated into a virtual reality environment, the chatbot interface also includes the function of displaying the relevant components or specification information of power transformers. The related information of the key components will be displayed in the dialogue window when users click on the key components. For example, if users want to know what the cooling system is, they can simply move the mouser on the equipment and the specifications and model of the cooling system will be immediately displayed in dialogue window. In this way, users can conduct engineering consultation within the chatbot interface and retrieve information.

In the second sub-system, due to the large number of equipment and materials in power transformers, a structured database is needed to store information in a knowledge base. The information of the knowledge base uses the MySQL database server. The information includes raw materials, specifications, unit cost of key components stored in the MySQL server that can be passed back to the Unity environment of the chatbot to generate a bill of materials (BOM) table and presents tentative cost using a .Net Framework package.

\section{Implementation}

The system for this provides real-time engineering consultation, automatically generated bill of materials (BOM) for cost estimation when receiving an RFQ summary file, and provides user an immersive environment to interact with $3 \mathrm{D}$ models. This section discusses the implementation of the system including the introduction of functions and interface design of the question answering system (chatbot). Included in the discussion is an overview of the virtual reality environment, output of cost estimations, scene designs, transformer models construction and illustrations of user interaction in an immersive environment.

\subsection{Chatbot development}

The system provides an interface in which users can easily ask questions (in plain English), and receive a concise and insightful answer in real-time. The chatbot also integrates speech recognition, allowing users to communicate directly with the system through voice input. The question-answer dataset used in this system comes from the public FAQs on the webpages of different transformer suppliers. The answering capability of consultation chatbot depends on the completeness and correctness of the FAQ database. Therefore, the collection of the FAQ database is a crucial factor. The questions include many types, including transformer knowledge, and customer support consultation. Table 2 provides examples of the FAQ database. 
Table 2. The sample FAQs for chatbot retrievals.

\begin{tabular}{lll}
\hline \multicolumn{1}{c}{ Question } & \multicolumn{1}{c}{ Answer } \\
\hline Transformer & What is material of transformer core ? & $\begin{array}{l}\text { A transformer core is made from many sheets } \\
\text { if special steel to reduce losses and.... }\end{array}$ \\
\hline $\begin{array}{l}\text { International } \\
\text { Standard }\end{array}$ & $\begin{array}{l}\text { What is the international standard we } \\
\text { should choose? }\end{array}$ & $\begin{array}{l}\text { In accordance with the IEEE standard design } \\
\text { will be the most economical ... }\end{array}$ \\
\hline $\begin{array}{l}\text { Customer } \\
\text { Service }\end{array}$ & How long does installation take? & General 6-8 weeks \\
\hline $\begin{array}{l}\text { Engineering } \\
\text { Knowledge }\end{array}$ & What is induced electricity? & $\begin{array}{l}\text { Electricity generated by relative motion of a } \\
\text { magnetic field and an electric charge. }\end{array}$ \\
\hline
\end{tabular}

Retrieval-based chatbots rely on a complete and systematic database construction. This research enters 100 questions into the chatbot dataset to evaluate the accuracy rate of the question answering system. The questions are collected from a website called Quora. All the questions and answers in Quora are created, edited, and organized by the people who use it. The source of the database used in this research is the FAQs from engineering manufacturers, the testing questions can be used to test whether the chatbot system can answer the public's questions about the transformer and further improve or update the database. From the results of testing, there are 14 wrong answers out of 100 questions tested, with an accuracy rate of $86 \%$.

\subsection{Output of cost estimation system}

This section introduces the result of the second sub-system of the research. Based on previously mentioned processes of the cost estimation system, the user is required to upload a file of the RFQ summary into the proposed system. The cost estimation system will compare each component of the RFQ summary with the KB and check if all of the components satisfy an output which includes all key components within estimated costs. The partial output of the cost estimation system is shown in Figure 5. The system extracts the key components from the RFQ summary which the user provides, such as copper wire, type of insulating oil, number of fans, and the type of winding.

This information is critical to the design of the transformer and determines the cost. Furthermore, the supplier of transformers has the tentative costs estimation report which helps to decrease the time of processing an order. This enables manufacturers and customers to be more efficient in establishing a reasonable price.

\begin{tabular}{|c|c|c|c|c|c|}
\hline No. & Name & Quantity & Unit & Spec & Cost \\
\hline 1 & Copper Wire & 1067.25 & $\mathrm{KG}$ & $325 \mathrm{~mm}$ & $218,788.00$ \\
\hline 2 & Tape & 9.00 & ROLL & P-G 50W & $57,162.00$ \\
\hline 3 & Insulating Tube & 1.00 & SET & - & $131,892.00$ \\
\hline 4 & On Load Tap Changer & 1.00 & SET & - & $2,614,742.00$ \\
\hline 5 & Latch R & 1.00 & PIECE & SIEMENS & $9,143.00$ \\
\hline 6 & Electric Wire & 5000.00 & FOOT & SIS/XHHW-2 & $55,483.00$ \\
\hline 7 & Insulating Oil & 34064.00 & $\mathrm{~L}$ & NAD & $1,076,868.00$ \\
\hline 8 & Fan & 6.00 & PIECE & - & $155,102.00$ \\
\hline 9 & Oil Level Gauge & 1.00 & SET & QUALITROL & $17,250.00$ \\
\hline 10 & Silicon Steel Plate & 14719.00 & KG & F27D090 & $1,349,168.00$ \\
\hline.. &.. &.. &.. &.. &. \\
\hline$\ddot{~}$ & .. & .. & . & .. &. \\
\hline & & & & Total Cost : & $13,842,114.00$ \\
\hline
\end{tabular}

Figure 5. The example output of BOM cost estimation module. 


\subsection{VR-enabled transformer mass-customization demonstration}

This section introduces the implementation of the QA system and the cost estimation system within the immersive environment. In addition to the abilities of question answering and cost estimation, the proposed system can provide an immersive environment that the user interacts with. Users can interact with the $3 \mathrm{D}$ models using a keyboard or mouse without the virtual reality hardware. The other platform enables users to interact with the consultation system using a helmet and controller in the immersive environment. The following section focuses on introducing scenes design and objects control in the constructed immersive environment.

According to the customer's requirements, the environment of presenting a power transformer model can be divide into two situations: indoor and outdoor. As shown in Figure 6 (both outdoor and indoor scenes), the outdoor environment simulates a transformer built in a desert. In the real-world issues such are "how to outlay the electric wire" and "how to determine the precise position of the transformer" must be considered during design. The VR chatbot can help the manufacturer and customer increase the efficiency of the design process. Another scene simulates a large transformer set in a building, which has space limitations including the scale, vibration, and noise of the transformer. Consequently, the VR system can provide an environment which helps the user or manufacturer clearly understand the transformer installation.
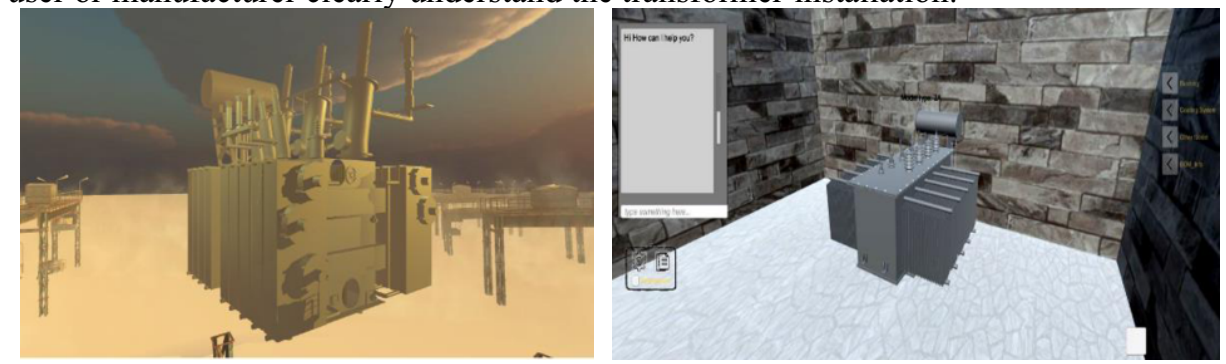

Figure 6. The outdoor and indoor transformer scenes.

In addition to the presentation of the model and the environment, the VR system can interact with the user in real time. Figures 7 presents the animation of transformer disassembly. Users can control the events by saying a specific word. For example, the user can say: "disassemble" to the system. Within two seconds, when the speech recognition system receives this command, it will generate the model disassembly animation. By disassembling transformer, the user can better understand the details of components and structure. This also helps the manufacturer to provide a better visual presentation when introducing products to customers. Another function of the immersive environment is transformer hyalinization. Users can control the events by using the controller or mouse to trigger the button. The transparency of the transformer will gradually increase when users click the button. Using transformer hyalinization, users can obviously see the detail of coil and inner structure. As discussed before, power transformers are highly customized products. The VR system provides the function of replacing transformer components or selecting other transformer models. As shown in Figure 7, users can change components or models by using the controller or the mouse to interact with system. By introducing these functions, users can select the components or models which they want and further acquire the related information, which can increase efficiency of the communication. 


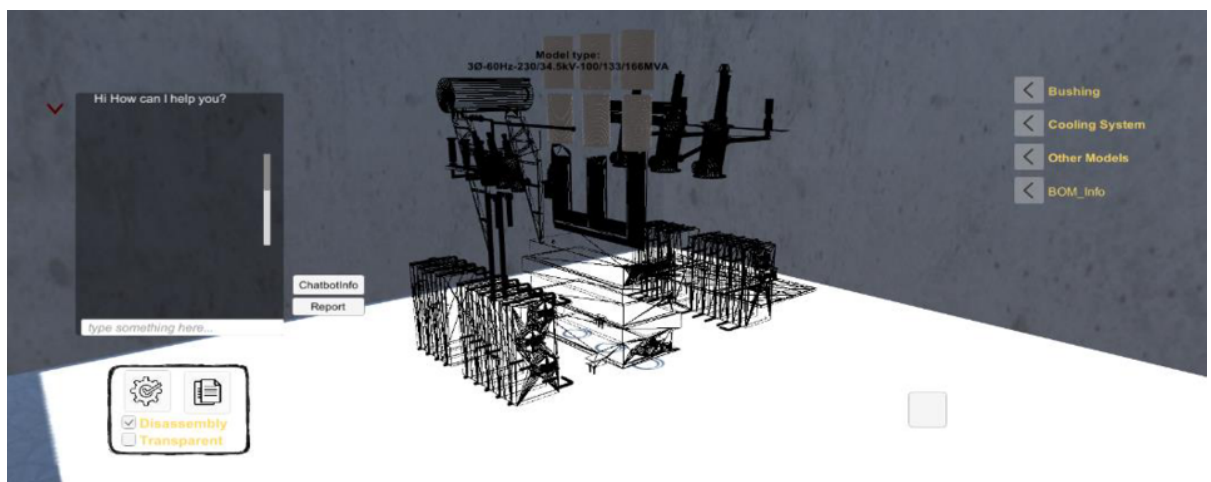

Figure 7. The transformer disassembly for detailed design review.

\section{Conclusion}

This research proposes a system framework of a chatbot that integrates Q\&A system into a VR environment. In addition to providing engineering consultation, the system also combines the advantages of VR to provide a user 3D model interface which helps improve understanding of complex transformer designs. The contribution of this research is to propose a VR enabled engineering consultation chatbot that users can interact with simultaneously with an immersive environment. This allows for users to ask technical questions, obtaining tentative costs, while interacting with 3D models for vivid design reviews for mass customization of complex products.

\section{References}

[1] D. Jurafsky and J. H. Martin, Speech and Language Processing, 2nd ed. Prentice Hall, Boston, 2018

[2] A. Deshpande, A. Shahane, D. Gadre, M. Deshpande4, and P. M. Joshi, A survey of various chatbot implementation techniques, International Journal of Computer Engineering and Applications, 11, 2017, pp. 320-330.

[3] A. Mishra, S. K. Jain, A survey on question answering systems with classification, Journal of King Saud University-Computer and Information Sciences, 2016, 28(3), pp. 345-361.

[4] Y. Wu, W. Wu, C. Xing, Z. Li, and M. Zhou, Sequential Matching Network: A New Architecture for Multi-turn Response Selection in Retrieval-Based Chatbots, Computational Linguistics, 2016, 45 (1), pp. 163-197.

[5] N.J. Hong, U. H. Govindarajan, Y. C. Chang Chien, Amy J.C. Trappey, Comprehensive Technology Function Product Matrix for Intelligent Chatbot Patent Mining, 2019 IEEE International Conference on Systems, Man, and Cybernetics (SMC), 2019, DOI: 10.1109/SMC.2019.8914365.

[6] G.C Burdea and C. Philippe, Virtual Reality Technology, 2nd ed. John Wiley \& Sons, New York, 2003.

[7] A. Y. C. Nee, and S. K. Ong, Virtual and Augmented Reality Applications in Manufacturing, IFAC Proceedings Volumes, 46(9), pp. 15-26, 2013.

[8] N. S. Hamid, F. A. Aziz, and A. Azizi, Virtual reality applications in manufacturing system, Science and Information Conference, 2014, DOI: 10.1109/SAI.2014.6918317.

[9] G. Gonzalez-Badilloa, H. I. Medellin-Castilloa, T. Lim, Development of a Haptic Virtual Reality System for Assembly Planning and Evaluation, Procedia Technology, 2013, Vol. 7, pp. 265-272.

[10] G. Peng, G. Wang, W. Liu, and H. Yu, A desktop virtual reality-based interactive modular fixture configuration design system, Computer-Aided Design, 2010, 42(5), pp. 432-444. 